\title{
Quartz Crystal Microbalance Humidity Sensor with Porous Electrodes
}

\author{
Kosmas Galatsis, Wenmin Qu and Wojtek Wlodarski \\ Sensor Technology Laboratory, Department of Communication and Electronic Engineering, \\ RMIT University, Victoria 3001, Melbourne, Australia
}

\begin{abstract}
This short paper describes a new idea for humidity measurement using QCM (Quartz Crystal Microbalance) devices. It is based on an etching approach which produces micropores into the electrodes of a QCM, instead of the common approach of coating the electrodes with hygroscopic materials. The electrodes are etched wet-chemically using buffered king water $\left(\mathrm{NHO}_{3}: \mathrm{HCl}=1: 3\right)$. The electrode surface becomes rough, resulting in a large surface area for water adsorption. Photolithographic etching using a plasma will be the next research stage, this will eliminate the generation of a randomly dented surface, hence providing a uniform porous structure. Fabrication and results of preliminary measurement are described in this paper.
\end{abstract}

\section{INTRODUCTION}

Quartz Crystal Microbalance (QCM) gravimetric devices are very attractive for humidity sensing applications as they employ the most basic physical effect which occurs when water molecule adsorption takes place on their surface. Sensors based on QCM exhibit superior noise immunity, exceptional temperature insensitivity and long term stability [1-3]. In addition, the sensor provides a direct frequency output signal, which is quasi-digital.

The first use of QCM for humidity sensing was reported by King in 1966, employing Au, Ni, and Al electrodes [4]. The sensitivity was relatively low due to the inert nature of $\mathrm{Au}, \mathrm{Ni}$, and $\mathrm{Al}$. Since then, many types of hygroscopic materials have been used, such as films of $\mathrm{SiO}_{x}$, gelatine and various kinds of polymers. These coatings improve the sensitivity dramatically, however, they simultaneously lead to a poor selectivity as some hygroscopic materials interact with other gaseous substances in the medium. Furthermore, coated polymers can frequently exhibit poor long-term stability.

This paper describes a new technique for improving the sensitivity of a QCM humidity sensor. It is based on an etching approach which "digs" micropores into the electrodes of a QCM, instead of the common approach of coating the electrodes with hygroscopic materials. Electrodes were wet-chemically etched using buffered king water $\left(\mathrm{NHO}_{3}: \mathrm{HCl}=1: 3\right)$ which caused the electrode surface to become jagged. Not only does a rough surface increase the surface area for water adsorption, but more importantly, it provides the possibility for capillary condensation of water within the pores, increasing the surface area and hence enhancing the sensors humidity sensing properties.

\section{CONCEPT OF NEW METHODOLOGY}

The sensitivity of a QCM sensor depends on the mass change of the crystal resonator. Assuming the mass change $\Delta m$ is much smaller than the electrode mass $m$, the frequency change $\Delta f$ is influenced by a mass change on the electrodes of the crystal [5]:

$\Delta f=-f_{0} \frac{\Delta m}{m}$

where $f_{0}$ is the natural oscillating frequency of the crystal, being commonly an AT-cut quartz, vibrating in its thickness shear mode.

For a humidity sensor, the sensitivity depends on the amount of water adsorbed on the surface of the QCM. Adsorption of water vapour on the surface of metals, metal oxides and even plastics is a common phenomena. In any case, adsorbed water molecules increase the conductivity, dielectric constant and the mass of the sensing material [6]. When good conductive metals are used as sensor materials, the humidity can not be measured in terms of resistance change or capacitance change. However, the humidity change can be measured using a QCM device.

Generally, water adsorption at a metal oxide can be divided into three stages: chemisorption, physisorption and capillary condensation. At a low humidity water vapour chemisorbs by a dissociation mechanism, forming hydroxyl ions at the surface [7]. With increased humidity, subsequent layers of water molecules will be physically adsorbed on this chemisorbed layer, as shown in Fig. 1. With a further increase in humidity, the multi-layers of physisorbed water molecules tend to condense in capillary pores with a radius below the Kelvin radius $\left(r_{k}\right)$, which is defined as [8]:

$$
r_{k}=\frac{2 \jmath M}{\rho R T \ln \left(P_{s} / P\right)}
$$


where $\gamma$ is the surface tension of water, $M$ is the molecular mass of water, $\rho$ is the density of water, $P$ is the vapour pressure and $P_{s}$ is the vapour pressure at saturation.

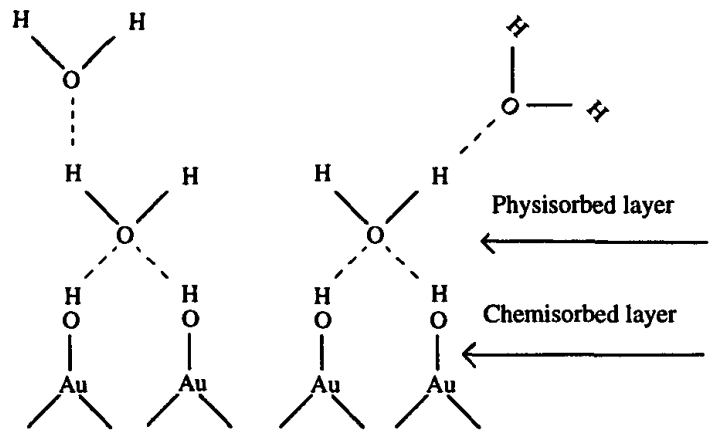

Fig. 1. Schematic representation of water absorption on a solid state surface.

It is believed that water adsorption on a Au-surface occurs only by chemically and physically means, since the Ausurface is relative well defined with no capillary pores. The primary feature of this new methodology is to increase water adsorption by the use of microstructuring technology capable of etching micropores on Au-surfaces, instead of traditionally coating the surface using hygroscopic humidity sensing materials. The humidity sensitivity could be enhanced by the water adsorbed in the pores. Fig. 2 illustrates this new concept.

From the technical point of view, it is possible to create Cr-masks for UV-lithographic etching with transparent holes of diameters less than $0.5 \mu \mathrm{m}$. Dry etching techniques like plasma etching, RIE (Reactive Ionic Etching), or excimer lasers are also feasible for creating micropores. However, before the relatively expensive photolithographic processing approach is undertaken, a feasibility study using maskless, time controlled wet-chemical etching using buffered king water requires to be undertaken.

\section{EXPERIMENTAL}

Commercially available AT-cut QCMs with Aumetallisation (Bright Star Pty Ltd, Australia) have been used. The Au-electrode was wet-chemically etched using a maskless time controlled process emptying buffered king water. $\mathrm{Cu}$-wires are connected using epoxy to etched quartz wafer for the measurement, as shown in Fig. 3. The humidity response of the QCM was obtained using a Keithley 775A frequency counter.

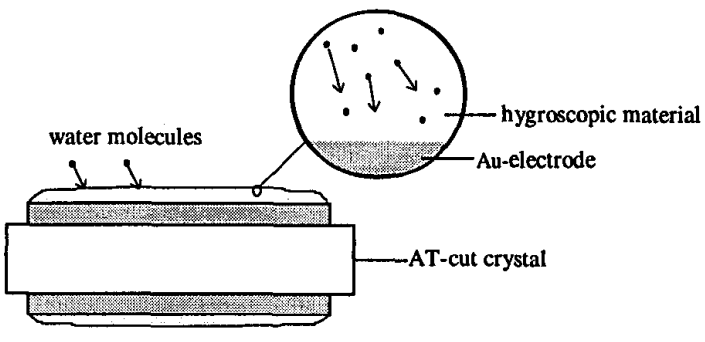

(a)

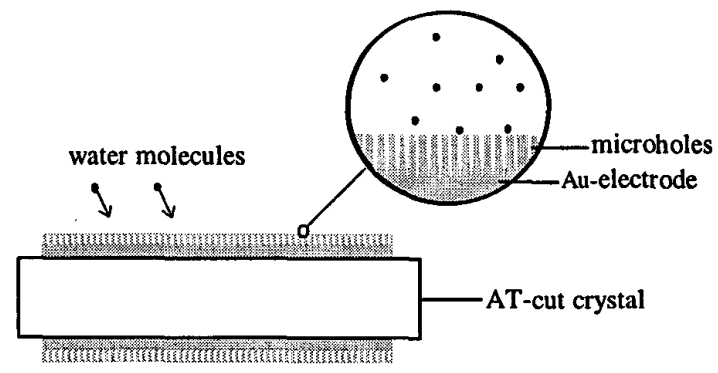

Fig. 2. Traditional QCM sensor for humidity detecting(a) and new concept of the QCM humidity sensor(b).

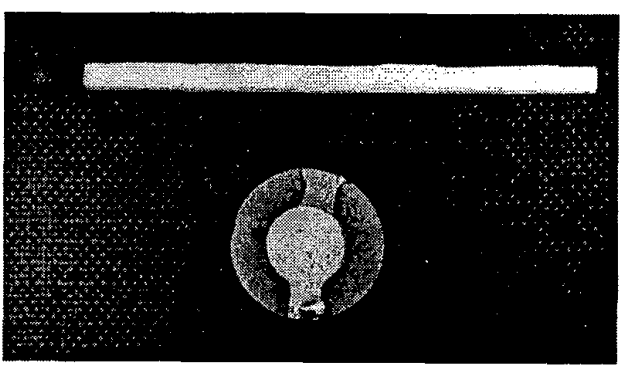

Fig. 3. Photography of QCM humidity sensor.

The humidities are generated using satisfied salts. The humidity at a temperature of $25{ }^{\circ} \mathrm{C}$ are given in the following table 1 .

Table 1. Humidity values of different satisfied salts.

\begin{tabular}{lcl}
\hline Salt & & Humidity \\
\hline $\mathrm{LiCl} \bullet \mathrm{H}_{2} \mathrm{O}$ & (Lithium Chloride) & $12 \%$ \\
$\mathrm{MgCl}_{2} \bullet 6 \mathrm{H}_{2} \mathrm{O}$ & (Magnesium Chloride) & $33.2 \%$ \\
$\mathrm{Mg}\left(\mathrm{NO}_{3}\right)_{2} \bullet 6 \mathrm{H}_{2} \mathrm{O}$ (Magnesium Nitrate) & $53.4 \%$ \\
$\mathrm{NaCl}$ & (Sodium Chloride) & $75.8 \%$ \\
$\left(\mathrm{NH}_{4}\right)_{2} \mathrm{SO}_{4}$ & (Ammonium Sulphate) & $80.0 \%$ \\
$\mathrm{KNO}_{3}$ & (Potassium Nitrate) & $92.0 \%$ \\
$\mathrm{~K}_{2} \mathrm{SO}_{4}$ & (Potassium Sulphate) & $96.9 \%$ \\
\hline
\end{tabular}




\section{RESULTS AND Discussions}

SEM (Scanning Electron Micrography) examination reveals a rough surface of the Au-electrode after wetchemically etching. The rough Au-electrode increases the surface area for water adsorption and more importantly, providing micropores for water condensation. The humidity-sensitivity is greatly improved by the increased water adsorption, as shown in Fig. 4. The upper part of the pores (where the radius is greater) have no affect on the sensitivity, however, they play a vital role in water transportation, which is important for a fast response.

This initial experiment conformed that our concept works. The wet-chemically etched Au-electrode is however, random. To obtain a sensor with a good reproductivity, radomness has to be eliminated. To eliminate such randomness it would be desirable to fabricate a well-defined porous structure with a controllable and predictable etching technique such as photolithography. This maybe the next step of our work.

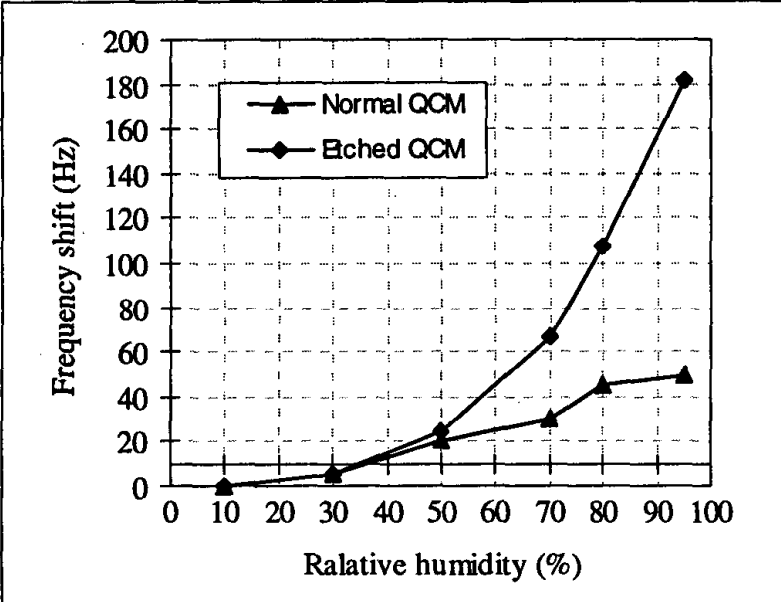

Fig. 4: Sensitivity comparison of a standard and an etched $10 \mathrm{MHz}$ QCM sensor.

\section{CONCLUSION}

It has been shown by wet-chemically etching using a maskless, time-controlled process employing buffered king water the electrode surface becomes rough resulting in a large surface area for water adsorption and hence increasing the humidity sensing properties of a QCM. Concluding, to complete this study and reduce the random generating porous creation it has been proposed to use photolithography technology.

\section{REFERENCE}

[1] W. Goepel, J. Hesse and J. N. Zemel Sensors: A Comprehensive Survey. 9. Bd. Weinheim: VHC Verlagsgesellschaft 1994.

[2] A. Wajid, "On the accuracy of the quartz-crystal microbalance (QCM) in thin-film depositions" Sensors and actuators a 63 (1997), pp. 41-46.

[3] D. Salt, Hy-Q handbook of quartz crystal devices. Van Nostrand, UK, 1987

[4] King, Jr. W. H. Research and development Vol. 20 No. 4 (1969) pp. 28-34.

[5] M. Rodahl and B. Kasemo, "On the measurement of the thin liquid overlayers with the quartz-crystal microbalance, Sensors and Actuators A54, 1996, pp. 448-456.

[6] E. Traversa, "Ceramic sensors for humidity detection: the state-of-theart and future developments", Sensors and Actuators B 23, 1995, pp. 135-156.

[7] W. Qu and J.-U. Meyer, "A novel thick-film ceramic humidity sensor". Sensors and Actuators Vol. B40, No. 2 - 3 (1997) pp. 175182.

[8] G. M. O'Halloran, M. Kuhl, P.J. Trimp and P.J. French, "The effect of additives on the adsorption properties of porous silicon'Sensors and Actuators A 61, 1997, pp. 415-420. 